\title{
The Neurobiology of Autism Spectrum Disorder As It Relates to Twice Exceptionality
}

\author{
Rachel J. Sharkey ${ }^{a, b}$ \& Thomas Nickl-Jockschat ${ }^{a, b, c}$ \\ alowa Neuroscience Institute, Carver College of Medicine, University of lowa, lowa City, IA, USA \\ ${ }^{b}$ Department of Psychiatry, University of lowa, lowa City, IA, USA \\ 'Department of Neuroscience and Pharmacology, Carver College of Medicine, University of lowa, \\ lowa City, IA, USA
}

Corresponding author:

Rachel J. Sharkey: rachel-sharkey@uiowa.edu

Keywords: Twice exceptionality, Autism spectrum disorder, Neuroimaging, MRI

\begin{abstract}
There is a long-standing association between exceptional cognitive abilities, of various sorts, and neuropsychiatric illness, but it has historically largely been investigated in an exploratory and non-systematic way. One group in which this association has been investigated with more rigor is in subjects who have been identified as twice exceptional; an educational term describing subjects who are both gifted and diagnosed with a neuropsychiatric disability. This term covers multiple conditions, but is of specific interest in particular in the study of autism spectrum disorder. Recent findings have led to the development of a hypothesis that a certain degree of the neurobiology associated with autism might even be advantageous for individuals and could lead to high giftedness, while becoming disadvantageous, once a certain threshold is surpassed. In this model, the same neurobiological mechanisms confer an increasing advantage up to a certain threshold, but become pathological past that point. Twice-exceptional individuals would be exactly at the inflection point, being highly gifted, but also symptomatic at the same time. Here, we review how existing neuroimaging literature on autism spectrum disorder can inform research on twice exceptionality specifically. We propose to study key neural networks with a robust implication in ASD to identify the neurobiology underlying twice-exceptionality. A better understanding of the neural mechanisms of twice exceptionality should help to better understand resilience and vulnerability to neurodevelopmental disorders and to further support affected individuals.
\end{abstract}




\section{Introduction}

There is a long-standing and frequently established correlation between intellectual giftedness and creativity, and neuropsychiatric disease (Andreasen \& Canter, 1974; Crespi, 2016; Gardner et al., 2020). This relationship was first formally identified by Andreasen et al. based on studies of the participants in a highly competitive Creative Writing Masters of Fine Arts degree program. Gifted writers were found to have significantly more relatives with neuropsychiatric illness and showed increased rates of neuropsychiatric symptoms, although not necessarily diagnoses, compared to controls (Andreasen \& Canter, 1974). The relationship between these traits appears counterintuitive, and there is currently not an established explanation for the co-occurrence of increased ability and potentially debilitating neuropsychiatric dysfunction. One potential interpretation is that the same neurobiological changes are associated with both these conditions in an inverse- $U$ relationship. In this model the same mechanism or group of mechanisms confers an increasing advantage up to a certain threshold, but becomes pathological past that point (Figure 1).

While an overall relationship between highly creative and intellectual subjects and a family history of neuropsychiatric illness has been established, prior studies have largely been small and highly exploratory. These studies also utilize a wide variety of definitions of both ability and neuropsychiatric illness. In order to study this relationship systematically a more rigorous definition is needed. So-called twice exceptional individuals, first identified in educational studies of giftedness, constitute a clearly defined group expressing both increased cognitive abilities and neuropsychiatric disorders (Andreasen \& Canter, 1974; Assouline et al., 2012; Cain et al., 2019).

\section{Twice-Exceptionality}

Twice exceptionality is defined as the combination of intellectual giftedness, and a psychiatric or neurodevelopmental disorder (Cain et al., 2019). The twice-exceptional population is small, but unlike the cohorts used in the creativity studies, the twice-exceptional population is relatively rigorously defined and - by definition - linked to psychopathology. This is an improvement on the creativity cohorts in two ways, from an experimental perspective; firstly, it allows for an operational definition of twice-exceptionality, and, secondly, twice exceptional cohorts can be meaningfully placed on distinct scales (according to IQ or other measures of cognitive function) to quantify their relationship with control cohorts.

Twice exceptional individuals represent a unique opportunity to explore the hypothesis of a continuum between giftedness and neuropsychiatric disease, since they may indicate exactly the inflection point where neurobiological change moves from advantageous to pathological (Figure 1). Twice-exceptional individuals exhibit both advantage and pathology simultaneously. Delineating the neural correlates of giftedness and how those correlates change in twice exceptional individuals who exhibit neuropsychiatric illness at the same time could provide a decisive model of mechanisms contributing to vulnerability and resilience to neuropsychiatric illness. Brain research is also a much needed avenue of support for twice exceptional individuals 
who are not well represented in current neuroscience research and who may require different supports than both other gifted students and other students with psychiatric or neurodevelopmental conditions (Assouline et al., 2012; Cain et al., 2019).

Understanding the neural basis of twice-exceptionality involves approaching it from two directions: establishing firstly how twice-exceptional individuals differ from their neurotypical and gifted peers, and secondly how they differ from non-twice-exceptional peers with the same diagnoses. To date, twice exceptionality has been primarily investigated in the context of education, meaning that our current understanding of the neural basis of twice-exceptionality remains sparse. Twice-exceptionality covers a wide range of conditions, but autism spectrum disorder (ASD) is a commonly studied diagnosis for individuals who have been identified as twiceexceptional (Cain et al., 2019; Doobay et al., 2014). Existing research on ASD also provides a useful framework for approaching twice-exceptionality research from the second direction; establishing which neural features twice exceptional subjects with ASD share or do not share, with other ASD sub-populations. There is a well-developed pre-existing literature on neuroimaging findings associated with ASD which can provide the basis for developing specific hypotheses about the neuroanatomical features of twice exceptional ASD. ASD has been associated with alterations to the overall trajectory of brain growth, and also with structural and functional changes in key networks that are likely associated with core symptoms, namely the face processing network, striatal networks, and the corpus callosum (Chang et al., 2021; Fuccillo, 2016; Nomi \& Uddin, 2016; Sui et al., 2018). It is likely that disturbed trajectories of brain development result in perturbations of the connectivity of large-scale networks (Coupé et al., 2017; Courchesne et al., 2003; Redcay \& Courchesne, 2005). These three networks could all be potentially valuable targets for researching the neural correlates of twice exceptionality. In the following, we will review the evidence for the involvement of these networks in ASD pathophysiology and discuss the potential relevance for twice exceptionality.

\section{Variation in Cognitive Abilities Within the ASD Population}

ASD is a pervasive developmental disorder defined by persistent deficits in social interaction and behavior and communication, as well as restricted interests and repetitive patterns of behavior (DSM-V Task Force, 2013). In addition to its core symptoms, ASD is associated with other deficits including problems with motor skills, and, in a significant number of cases, intellectual disability (ID). The association between ASD and ID is strong, but there is also evidence that it is inflated by diagnostic bias. Older studies have found that up to $70 \%$ of patients diagnosed with ASD also had comorbid ID. More recent, larger studies using a more aggressive community sampling method found a lower, although still substantial rate of closer to $50 \%$. There is also evidence that the rate of ASD diagnoses in patients without ID is increasing faster than the diagnosis in patients with both ASD and ID, which may indicate that ASD in patients with normal or elevated IQ may be underdiagnosed compared to patients with comorbid ID (Charman et al., 2011; Matson \& Shoemaker, 2009; Riedel et al., 2014). Despite the strength of this correlation, however, patients with ASD do represent the full spectrum of intellectual abilities, including those diagnosed as twice exceptional. Some prior research seeking to differentiate between ASD patients with and without ID has focused on so-called "high-functioning ASD", a broad category which includes any 
patients with both ASD and an IQ greater than 70 (and therefore not meeting the criteria for a diagnosis for ID), and "low-functioning ASD", those patients with a comorbid ID diagnosis.

Genetic research has also identified differences in heritable risk between high and low functioning subpopulations with ASD. A large study based in Sweden examining the relationship between paternal IQ and risk for neurodevelopmental disorders in children found that there was a significant association between high paternal IQ and risk for offspring with high functioning ASD, but not offspring with low functioning ASD (Crespi, 2016; Gardner et al., 2020). This suggests that these two groups have only partially overlapping genotypes (Moreau et al., 2021; Rein \& Yan, 2020).

\section{Neuroimaging Findings In ASD}

Symptoms of ASD present early in development and persist across the lifespan. This indicates that the etiology of ASD likely originates in differences beginning early in neurodevelopment. Brain development is a continuous process which begins during early fetal development and continues throughout the lifespan. Brain development is also not a single, unified process. Different regions of the brain have distinct developmental trajectories, resulting from the intricate interplay of multiple biological processes, involving, e.g., cell migration and differentiation, as well as the formation and elimination of synapses (Coupé et al., 2017; Shin et al., 2018).

There is a growing body of evidence that the trajectories of overall brain growth are disrupted in ASD in a nonlinear fashion. At birth, head circumferences of children who are later with diagnosed with ASD are often smaller than average. In toddlers, head circumference correlates well with overall brain growth and can replace neuroimaging to a certain degree (Bartholomeusz et al., 2002). This is followed by a period of relative brain overgrowth, leading to an increase above average in head and brain size in young children with ASD. But this is followed by a reduction in growth in later childhood, resulting in an overall typical head size in adulthood (Courchesne et al., 2003; Redcay \& Courchesne, 2005). While this is a crucial piece of information for understanding the processes underlying the development of the brain in ASD, it also confirms that a simple model of under- or overgrowth of the brain as a whole will not be sufficient to explain the pathophysiology of ASD. Instead, a more complex model of how the trajectories of specific regions are altered in relation to the expected trajectory for typical development and to the trajectories of related regions is needed to understand how alterations in neurodevelopment ultimately give rise to persistent symptoms. This is difficult to accomplish, since high-resolution neuroimaging of regional trajectories relies heavily on magnetic resonance imaging (MRI). MRI has a much higher degree of spatial sensitivity than other human brain imaging modalities, but while this allows for the analysis of a high degree of anatomical detail, it is also very sensitive to subject motion which makes imaging of babies and toddlers logistically challenging (Ouyang et al., 2019). The literature of structural changes in ASD has therefore tended to focus on older ages, which more readily produces useable results, but misses the critical early development period entirely. MRI studies investigating developmental trajectories typically need to be large cohort 
studies and need specialized collection methods, if focused on early development. These are being developed, but remain a serious logistical challenge, especially when applied to a heterogenous population like children with ASD (Ouyang et al., 2019).

While the global volume of the brain appears to normalize in adulthood in ASD there is evidence of persistent differences in regional brain structural trajectories and connectivity between regions in ASD, which closely relate to ASD symptoms (Nickl-Jockschat et al., 2012; Janouschek et al., 2021; Nickl-Jockschat et al., 2015; Sui et al., 2018). Previous meta-analyses of structural and functional changes in ASD are oftentimes the only option to gather data from larger cohorts, as sample sizes of individual imaging studies oftentimes remain small due to the challenging recruitment of patients. These studies have implicated changes of structure and connectivity of regions with functions, which have obvious implications for the symptoms of ASD. However, the exact relationship between these regional structural and functional changes is also unclear. Structural and functional changes tend to co-occur in the same networks, but not at the same locations suggesting an overall pattern in which a disturbance in structure has downstream effects the functions of its connected networks. The exact mechanism by which this happens, however, merits further investigation (Nickl-Jockschat et al., 2015).

One of the key deficits associated with ASD is difficulty focusing on faces and extracting emotional cues from facial expressions. Atypical gaze is a very early indicator of ASD. Toddlers who go on to be diagnosed with ASD can be seen to look at human faces in videos less, and to visually track conversations less well than their typically developing peers (Chang et al., 2021). Face processing in neurotypical subjects involves the coordinated activity of a widely distributed face processing network comprised of distinct dorsal and ventral visual streams (Wang et al., 2020). A key region within that network is the fusiform gyrus. Patients with ASD were found to have both reduced activation in the left fusiform gyrus, a region heavily connected to a large number of other regions in the face processing network, including the lateral occipital, temporo-occipital, inferior frontal and parietal cortex, the thalamus and the amygdala. This network was also associated with structural changes in the occipital cortex, in V5, an early hub of basal motion processing that is included in the face processing network, and overlapped with networks involved in action observation (Nickl-Jockschat et al., 2015). The structural changes in early parts of the face processing network seem to have a downstream effect on the function and connectivity not only of the rest of the network, but potentially with associated networks as well. Some other studies have also found activation in alternate regions which may be further evidence of network disorganization (Nomi \& Uddin, 2016). How precisely the very early behavioral changes in gaze direction relate to the trajectory of development of V 5 in the early face processing network and then to the changes seen in imaging of older children and adults is similarly unclear.

A second region implicated in the pathophysiology of ASD is the basal ganglia. The basal ganglia are a highly conserved set of subcortical nuclei connected widely to the frontal cortex and the thalamus. They are involved in a diverse range of cognitive functions including reward processing, emotional regulation, goal-directed behavior, self-control, and motor planning. The functions of the basal ganglia have been widely implicated across multiple symptoms of ASD including 
abnormal reward processing, executive dysfunction, repetitive behaviour, and motor and sensory processing issues (Fuccillo, 2016). There is evidence that the different regions of the basal ganglia each have distinct developmental trajectories compared to both the cortex and other regions during typical development, making this another region where the established neuroimaging findings likely start early in development (Coupé et al., 2017). While disturbances of striatal circuits are admittedly not nosologically specific (Chase et al., Hum Brain Mapping, 2018), they seem to be a major hub mediating genetic vulnerability to ASD, as structural and functional changes associated with striatal networks can even be found in genetic mouse models of ASD (Angelakos et al., Autism Res, 2017; Grissom et al., Mol Psychiatry, 2018; Kumar et al., Transl Psychiatry, 2018). A recent meta-analysis identified hypoactivation in response to reward in the basal ganglia, focused in the right putamen and nucleus accumbens in subjects with ASD compared to controls. Importantly, this analysis also found that subjects with ASD demonstrated hypoactivation in response to both social and non-social rewards, demonstrating that reward processing as a whole, not only a specific social response, is altered in ASD. The functional changes identified were non-overlapping with, but adjacent to, regions of the basal ganglia which show structural changes in subjects with ASD, once again indicating another area in which structural changes in one area are related to functional changes in a wider network (Janouschek et al., 2021; Nickl-Jockschat et al., 2012).

Studies have, thirdly, implicated white matter pathologies in the pathophysiology of ASD. White matter - which is predominantly composed of myelinated axons, rather than the cell bodies, which predominate in grey matter - reflects the organization of the brain's connectivity more so than its regional activity. Specifically, the corpus callosum, the largest and most prominent white matter tract in the brain, seems to be affected. The corpus callosum connects the two hemispheres of the brain and is a primary pathway for interhemispheric integration. Studies comparing callosal structure in subjects with and without ASD found reduced white matter integrity specifically in the medial and posterior segments which predominantly connect the posterior lobes of the brain (Sui et al., 2018). Previous research has implicated the corpus callosum in determining cognitive processing speed; reduced processing speed is one of the most reliably identified cognitive deficits in ASD (Haigh et al., 2018; Sui et al., 2018). Sui et al. found that subjects diagnosed with ASD demonstrated both reduced processing speed and callosal white matter integrity compared to typically developing controls, and that callosal integrity in typically developing controls explained more than $70 \%$ of variance in processing speed. However, they failed to identify a significant correlation between callosal integrity and processing speed in subjects diagnosed with ASD suggesting that the relationship itself has been altered and raising the question of what alternate networks might predict processing speed in ASD (Sui et al., 2018). Future studies focusing on twice exceptional ASD will be able to build on previous research done in more general ASD cohorts. It remains to be determined whether decreased interhemispheric connectivity would lead to an improvement of monohemispheric processes, such as, e.g., verbal abilities, which are increased in some ASD subpopulations (Doobay et al., 2014). 


\section{The neurobiological basis of twice exceptionality: hints from neuroimaging studies}

Research comparing high- and low-functioning ASD cohorts have identified overlapping regions of structural change in the left inferior temporal gyrus in both ASD groups, but also identified more extensive structural change in the temporal lobe extending into the left middle temporal gyrus in low-functioning ASD than in high-functioning ASD (Cai et al., 2018). Older research examining differences between high-functioning ASD and the now-defunct category of Asperger's disorder also identified differences between these groups. Asperger's disorder was differentiated from autism by the lack of delays in language acquisition and problems with verbal communication, and explicitly excluded subjects with intellectual disabilities (DSM-IV-TR Task Force, 2000). High functioning ASD, in contrast, meets the same core diagnostic criteria as lowfunctioning ASD and is differentiated only by tested IQ. Subjects with Asperger's disorder were found to have intermediate overall grey matter volume between subjects with high-functioning ASD and controls in one study, and distinct patterns of volume reduction in the basal ganglia compared to subjects with high-functioning ASD in another (Lotspeich et al., 2004; McAlonan et al., 2008). The distinction between Asperger's disorder and autism is focused on language acquisition rather than $I Q$, and so is not entirely equivalent to twice exceptionality, but it does provide some evidence for subpopulations of ASD distinguished by cognitive phenotype having distinct neural endophenotypes (Lotspeich et al., 2004; McAlonan et al., 2008).

Neuroimaging and genetic differentiation between ASD with and without ID provide evidence that the two groups fall along a spectrum of affectedness likely involving multiple biological pathways. Differentiation between subjects with high-functioning ASD and Asperger's disorder, which is based on symptom burden rather than intellectual function, similarly suggests a spectrum of affectedness, although to integrate the two sets of data is not clear, especially since changes to diagnostic categories mean that these groups are no longer distinguished clinically.

In addition to the more widely used high versus low functioning comparisons, there is a small body of existing neuroimaging research focusing specifically on ASD patients with above-average IQ scores, which correspond much more closely to the definition of twice-exceptionality, although this research still is very scarce (Ecker, 2017; Riedel et al., 2014). This group is distinct from the more general high-functioning ASD population, because it draws on subjects with an ASD diagnosis and an above average tested IQ, whereas the high-functioning label applies to any subject without ID, which covers a range from the twice-exceptional one to below average, but above the threshold for a diagnosis of ID. It is also distinct from the definition of Asperger's Syndrome, which does not necessarily require an above average IQ. One study of high-IQ ASD subjects failed to identify significant differences between high IQ subjects with ASD and matched controls (Riedel et al., 2014). A second study, which used a slightly lower, but still above average IQ threshold of 110, found significant alterations in connectivity and trends towards reduced volume in the right temporoparietal junction and anterior temporal lobe compared to controls (Ecker, 2017). Taken together this pattern of results provides support for the idea that twice exceptionality represents an intermediate phenotype along a spectrum from increased ability to dysfunction, but it also suggests that neuroimaging studies of the twice exceptional phenotype 
need to be sufficiently powered to detect subtle changes, which can be challenging in neuroimaging cohorts.

Although these different sub-populations of subjects with ASD differ in levels of intellectual ability, they share a clinical phenotype related to their shared diagnosis, namely the core symptoms of ASD. Despite its limitations in describing twice exceptional ASD directly, existing research into the neural correlates of ASD can provide the basis for specific hypotheses for future studies about twice exceptional ASD. We would expect a shared endophenotype related to that clinical phenotype to be present across the full spectrum of intellectual ability in ASD. Regions commonly associated with ASD more generally can therefore provide the basis for studying twice exceptionality.

\section{Conclusion}

Prior neuroimaging research has identified a set of structural and functional endophenotypes associated with ASD involving the face-processing network, the basal ganglia and the corpus callosum, which are associated with core symptoms of ASD and seem to show persistent effects of alterations of regional developmental trajectories on brain function. Prior cognitive research characterizing twice exceptional subjects has identified a cognitive phenotype defined by above average verbal ability and below average processing speed, even in subjects with markedly elevated IQ scores (Figure 2). The association with processing speed is also a common phenotype within ASD, and is associated with the integrity of the corpus callosum more generally and suggests that the callosal phenotypes associated with ASD may be especially relevant to twice exceptionality. However, while there is evidence of common cognitive endophenotypes, which is suggestive of similarly shared neural endophenotypes, direct neuroimaging research on this population is extremely sparse. The existing body of neuroimaging literature in ASD provides ample basis for hypotheses about twice exceptional subjects, but to genuinely establish an understanding of twice exceptional ASD will require neuroimaging studies specific to this subpopulation. This will develop our understanding of this unique cohort, which should both deepen our understanding of the relationship between intellectual exceptionality and neuropsychiatric illness and also enable us to better provide for people who fall under the umbrella 
References

Andreasen, N. J. C., \& Canter, A. (1974). The creative writer: Psychiatric symptoms and family history. Comprehensive Psychiatry, 15(2), 123-131. https://doi.org/10.1016/0010440X(74)90028-5

Assouline, S. G., Nicpon, M. F., \& Dockery, L. (2012). Predicting the academic achievement of gifted students with autism spectrum disorder. Journal of Autism and Developmental Disorders, 42(9), 1781-1789. https://doi.org/10.1007/s10803-011-1403-x

Bartholomeusz, H. H., Courchesne, E., \& Karns, C. M. (2002). Relationship between head circumference and brain volume in healthy normal toddlers, children, and adults. Neuropediatrics, 33(5), 239-241. https://doi.org/10.1055/s-2002-36735

Cai, J., Hu, X., Guo, K., Yang, P., Situ, M., \& Huang, Y. (2018). Increased Left Inferior Temporal Gyrus Was Found in Both Low Function Autism and High Function Autism. Frontiers in Psychiatry, 9(October), 1-9. https://doi.org/10.3389/fpsyt.2018.00542

Cain, M. K., Kaboski, J. R., \& Gilger, J. W. (2019). Profiles and academic trajectories of cognitively gifted children with autism spectrum disorder. Autism. https://doi.org/10.1177/1362361318804019

Chang, Z., Di Martino, J. M., Aiello, R., Baker, J., Carpenter, K., Compton, S., Davis, N., Eichner, B., Espinosa, S., Flowers, J., Franz, L., Harris, A., Howard, J., Perochon, S., Perrin, E. M., Krishnappa Babu, P. R., Spanos, M., Sullivan, C., Walter, B. K., ... Sapiro, G. (2021). Computational Methods to Measure Patterns of Gaze in Toddlers with Autism Spectrum Disorder. JAMA Pediatrics, 27705, 1-10. https://doi.org/10.1001/jamapediatrics.2021.0530

Charman, T., Pickles, A., Simonoff, E., Chandler, S., Loucas, T., \& Baird, G. (2011). IQ in children with autism spectrum disorders: Data from the Special Needs and Autism Project (SNAP). Psychological Medicine, 41(3), 619-627. https://doi.org/10.1017/S0033291710000991

Coupé, P., Catheline, G., Lanuza, E., \& Manjón, J. V. (2017). Towards a unified analysis of brain maturation and aging across the entire lifespan: A MRI analysis. Human Brain Mapping, 38(11), 5501-5518. https://doi.org/10.1002/hbm.23743

Courchesne, E., Carper, R., \& Akshoomoff, N. (2003). Evidence of Brain Overgrowth in the First Year of Life in Autism. Journal of the American Medical Association, 290(3), 337-344. https://doi.org/10.1001/jama.290.3.337

Crespi, B. J. (2016). Autism as a disorder of high intelligence. Frontiers in Neuroscience, 10(JUN), 1-17. https://doi.org/10.3389/fnins.2016.00300

Doobay, A. F., Foley-Nicpon, M., Ali, S. R., \& Assouline, S. G. (2014). Cognitive, adaptive, and psychosocial differences between high ability youth with and without autism spectrum disorder. Journal of Autism and Developmental Disorders, 44(8), 2026-2040. https://doi.org/10.1007/s10803-014-2082-1

DSM-IV-TR Task Force, A. P. A. (2000). Diagnostic and statistical manual of mental disorders: $D S M-I V-T R$.

DSM-V Task Force, A. P. A. (2013). Diagnostic and statistical manual of mental disorders : DSMV. Fourth edition. Washington, DC: American Psychiatric Association, [1994] (C1994. https://search.library.wisc.edu/catalog/999733358502121

Ecker, C. (2017). The neuroanatomy of autism spectrum disorder: An overview of structural neuroimaging findings and their translatability to the clinical setting. Autism, 21(1), 18-21. 
https://doi.org/10.1177/1362361315627136

Fuccillo, M. V. (2016). Striatal circuits as a common node for autism pathophysiology. Frontiers in Neuroscience, 10(FEB). https://doi.org/10.3389/fnins.2016.00027

Gardner, R. M., Dalman, C., Rai, D., Lee, B. K., \& Karlsson, H. (2020). The Association of Paternal IQ With Autism Spectrum Disorders and Its Comorbidities: A Population-Based Cohort Study. Journal of the American Academy of Child and Adolescent Psychiatry, 59(3), 410-421. https://doi.org/10.1016/j.jaac.2019.04.004

Haigh, S. M., Walsh, J. A., Mazefsky, C. A., Minshew, N. J., \& Eack, S. M. (2018). Processing Speed is Impaired in Adults with Autism Spectrum Disorder, and Relates to Social Communication Abilities. Journal of Autism and Developmental Disorders, 48(8), 2653-2662. https://doi.org/10.1007/s10803-018-3515-z

Janouschek, H., Chase, H. W., Sharkey, R. J., Peterson, Z. J., Camilleri, J. A., Abel, T., Eickhoff, S. B., \& Nickl-Jockschat, T. (2021). The functional neural architecture of dysfunctional reward processing in autism. Neurolmage: Clinical, 31, 102700. https://doi.org/10.1016/j.nicl.2021.102700

Lotspeich, L. J., Kwon, H., Schumann, C. M., Fryer, S. L., Goodlin-Jones, B. L., Buonocore, M. H., Lammers, C. R., Amaral, D. G., \& Reiss, A. L. (2004). Investigation of Neuroanatomical Differences between Autism and Asperger Syndrome. Archives of General Psychiatry, 61(3), 291-298. https://doi.org/10.1001/archpsyc.61.3.291

Matson, J. L., \& Shoemaker, M. (2009). Intellectual disability and its relationship to autism spectrum disorders. Research in Developmental Disabilities, 30(6), 1107-1114. https://doi.org/10.1016/j.ridd.2009.06.003

McAlonan, G. M., Suckling, J., Wong, N., Cheung, V., Lienenkaemper, N., Cheung, C., \& Chua, S. E. (2008). Distinct patterns of grey matter abnormality in high-functioning autism and Asperger's syndrome. Journal of Child Psychology and Psychiatry, and Allied Disciplines, 49(12), 1287-1295. https://doi.org/10.1111/j.1469-7610.2008.01933.x

Moreau, C. A., Raznahan, A., Bellec, P., Chakravarty, M., Thompson, P. M., \& Jacquemont, S. (2021). Dissecting autism and schizophrenia through neuroimaging genomics. Brain, 144(7), 1943-1957. https://doi.org/10.1093/brain/awab096

Nickl-Jockschat, T., Habel, U., Maria Michel, T., Manning, J., Laird, A. R., Fox, P. T., Schneider, F., \& Eickhoff, S. B. (2012). Brain structure anomalies in autism spectrum disorder-a metaanalysis of VBM studies using anatomic likelihood estimation. Human Brain Mapping, 33(6), 1470-1489. https://doi.org/10.1002/hbm.21299

Nickl-Jockschat, T., Rottschy, C., Thommes, J., Schneider, F., Laird, A. R., Fox, P. T., \& Eickhoff, S. B. (2015). Neural networks related to dysfunctional face processing in autism spectrum disorder. Brain Structure and Function, 220(4), 2355-2371. https://doi.org/10.1007/s00429-014-0791-z

Nomi, J. S., \& Uddin, L. Q. (2016). Face processing in autism spectrum disorders: From brain regions to brain networks. Neuropsychologia, 176(1), 139-148. https://doi.org/10.1016/j.neuropsychologia.2015.03.029.Face

Ouyang, M., Dubois, J., Yu, Q., Mukherjee, P., \& Huang, H. (2019). Delineation of early brain development from fetuses to infants with diffusion MRI and beyond. Neurolmage, 185(October 2017), 836-850. https://doi.org/10.1016/j.neuroimage.2018.04.017

Redcay, E., \& Courchesne, E. (2005). When is the brain enlarged in autism? A meta-analysis of all 
brain size reports. Biological Psychiatry, 58(1), 1-9. https://doi.org/10.1016/j.biopsych.2005.03.026

Rein, B., \& Yan, Z. (2020). 16p11.2 Copy Number Variations and Neurodevelopmental Disorders. Trends in Neurosciences, 43(11), 886-901. https://doi.org/10.1016/j.tins.2020.09.001

Riedel, A., Maier, S., Ulbrich, M., Biscaldi, M., Ebert, D., Fangmeier, T., Perlov, E., \& Tebartz van Elst, L. (2014). No significant brain volume decreases or increases in adults with highfunctioning autism spectrum disorder and above average intelligence: A voxel-based morphometric study. Psychiatry Research - Neuroimaging, 223(2), 67-74. https://doi.org/10.1016/j.pscychresns.2014.05.013

Shin, J., French, L., Xu, T., Leonard, G., Perron, M., Pike, G. B., Richer, L., Veillette, S., Pausova, Z., \& Paus, T. (2018). Cell-specific gene-expression profiles and cortical thickness in the human brain. Cerebral Cortex, 28(9), 3267-3277. https://doi.org/10.1093/cercor/bhx197

Sui, Y. V., Donaldson, J., Miles, L., Babb, J. S., Castellanos, F. X., \& Lazar, M. (2018). Diffusional kurtosis imaging of the corpus callosum in autism. Molecular Autism, 9(1), 1-12. https://doi.org/10.1186/s13229-018-0245-1

Wang, Y., Metoki, A., Smith, D. V., Medaglia, J. D., Zang, Y., Benear, S., Popal, H., Lin, Y., \& Olson, I. R. (2020). Multimodal mapping of the face connectome. Nature Human Behaviour, 4(4), 397-411. https://doi.org/10.1038/s41562-019-0811-3 


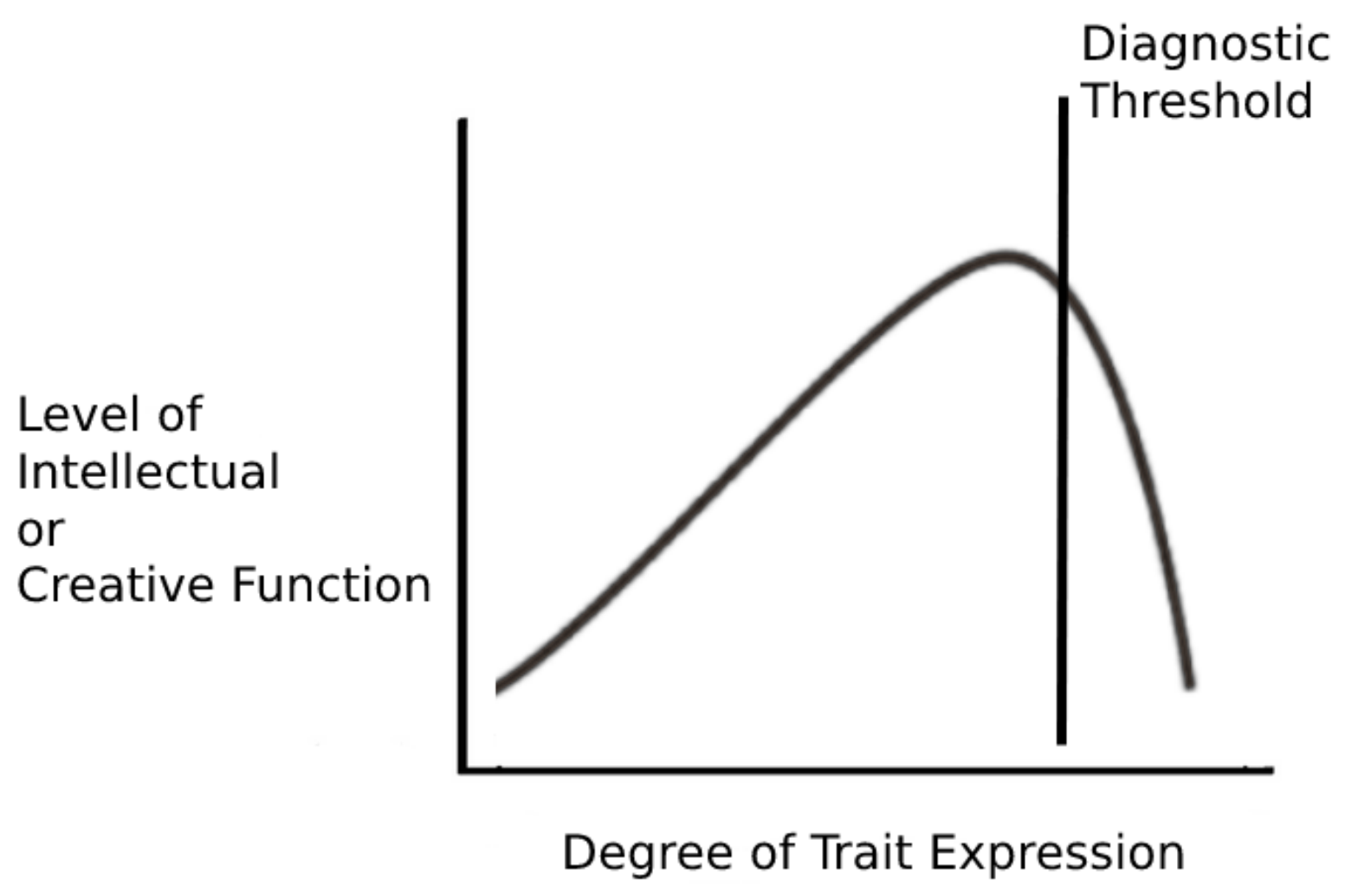

Figure 1. The proposed inverse- $U$ model of twice exceptionality. Increased trait expression increases cognitive ability up to a certain threshold, past which the relationship reverses and increased trait expression becomes pathological and reduces cognitive abilities. 


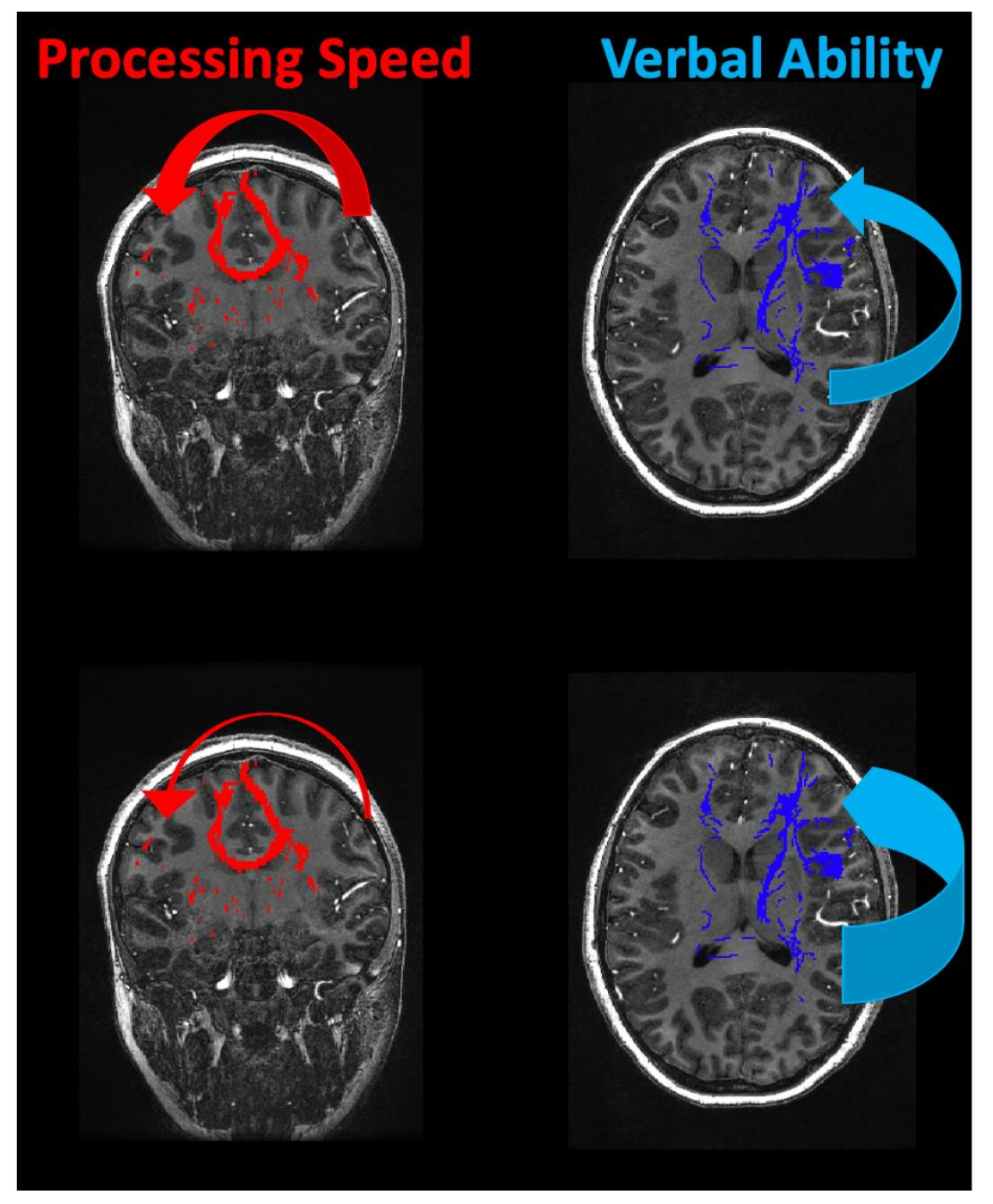

Figure 2. Cognitive studies of twice exceptionality have identified a common phenotype among subjects with twice exceptional ASD of above average verbal ability but below average processing speed, in subjects with above average IQ. Processing speed has been associated with the structural integrity of the corpus callosum in prior studies (red, left). Verbal ability is more often associated with the arcuate fasciculus (blue, right). 